\title{
Webinario: herramienta de integración en clases virtuales Webinar: Integration Tool in Virtual Class
}

\author{
José Bernardo Peńa Arcila ${ }^{1}$ y Nardis Coromoto Tovar Romero ${ }^{2}$ \\ Universidad de Granada, España \\ Hilda Rincón ${ }^{3}$ \\ Universidad Politécnica Territorial de Aragua. Venezuela
}

\section{RESUMEN}

Los seminarios web o webinarios, como instrumento académico utilizado por los estudiantes, tienen una innegable utilidad práctica. Además, cuentan con requerimientos básicos mínimos y objetivos valorables del programa académico. En este artículo se hurga en la relación que existe entre los webinarios y la forma en que los participantes incorporan esta herramienta a su sistema de aprendizaje emergente, complejo y colaborativo. Lo webinarios se mantienen en un ambiente virtual integrado con las aplicaciones informáticas, de manera que brinden los conocimientos técnicos en el área respectiva y permitan diseñar procesos colaborativos. Se analizó investigaciones previas que tuvieron un enfoque constructivista de la educación. El contexto estuvo determinado por 10 cursos de pregrado y postgrado de tres universidades durante 5 semestres; la población la conformaron 864 estudiantes de tecnología de la información y educación, en el estado Aragua de Venezuela. La investigación se centró en un estudio de caso realizado con un enfoque mixto, sustentado epistemológicamente en la teoría general de sistemas y la complejidad. En el enfoque cualitativo se empleó como técnica la entrevista a informantes claves, la autobiografía y el relato de experiencias durante el desarrollo del estudio. En el enfoque cuantitativo se utilizó la técnica de la encuesta, que recogió la percepción y valoración de los usuarios. El resultado permitió develar que más del $56 \%$ de sujetos se vieron motivados a realizar actividades académicas y fue poca la necesidad de contar con un tutor $(49,8 \%)$ para el esclarecimiento de las dudas que se les presentó; se generó un escenario de aprendizaje colaborativo. Además se comprobó la necesidad de que los tutores cambien de rol y de estrategias para este nuevo escenario.

Palabras Clave: Webinario, Clase virtual, Videoconferencia, Seminario web

\section{Abstract}

Web seminars or webinars, as an academic instrument used by students, have an undeniable practical utility. In addition, they have minimum basic requirements and valuable objectives of the academic program. This article explores the relationship between webinars and how participants incorporate this tool into their emerging, complex and collaborative learning system. The webinars

1 Doctor Profesor Titular Grupo de investigación TEIS. Universidad de Granada. España Email: josebernardo@ugr.es 
are maintained in a virtual environment integrated with computer applications, so that they provide the technical knowledge in the respective area and allow the design of collaborative processes. We analyzed previous research that had a constructivist approach to education. The context was determined by 10 undergraduate and postgraduate courses from three universities over 5 semesters; The population was formed by 864 students of information technology and education, in the Aragua state of Venezuela. The research focused on a case study carried out with a mixed approach, epistemologically supported in general systems theory and complexity. In the qualitative approach, we used the following techniques: interview to key informants, autobiography, and storytelling of experiences during study development. In the quantitative approach, the survey technique was used, which collected the perception and assessment of users. The result revealed that more than $56 \%$ of subjects were motivated to perform academic activities and there was little need to have a tutor $(49.8 \%)$ to clarify the doubts that they encountered; a collaborative learning scenario was generated. In addition, it was verified the need for tutors to change roles and strategies for this new scenario.

Keywords: Webinar, Virtual class, Videoconference, Web Seminar

\section{INTRODUCCIÓN}

La idea central del análisis que realiza este artículo es descubrir la relación entre los seminarios virtuales o webinarios y los estudiantes dentro del plan de trabajo académico en distintos cursos universitarios de pregrado y postgrado en varias universidades en el centro de Venezuela, con datos recogidos durante más de 2 años. Los estudiantes participantes se agruparon en equipos de 3 personas que prepararon un webinario relacionado con el objetivo del curso en el cual participaban.

Existen muchos debates y artículos que analizan las ventajas del webinario y sus aportes a las organizaciones en muchas áreas; pero en este caso se pretende reconocer cómo el ambiente colaborativo generado por esta herramienta motiva la participación de los estudiantes en los cursos académicos formales.

La incorporación de los webinarios al sistema de aprendizaje de los participantes debe estar valorada dentro de los objetivos del curso para así aumentar la motivación y creatividad en su utilización, pudiendo evitarse la apreciación subjetiva hacia un producto nuevo que pudiera sesgar las opiniones del grupo.

Todos los webinarios constan de una ponencia y un debate en línea donde los asistentes pueden preguntar y conversar con el experto. Según Vázquez (2011), estas nuevas formas de enseñanza no presencial no se limitan solo al aprendizaje autónomo en línea, se extienden también a otras metodologías de enseñanza colectiva habituales en las instituciones educativas, como seminarios, conferencias o talleres. Es posible desarrollar este tipo de formación virtual gracias a un software especializado que permite la asistencia conjunta $\mathrm{y}$ en directo de un amplio grupo de alumnos a sesiones formativas en las tiene la posibilidad de participar en forma activa. Además, tanto la ponencia como el debate pueden seguirse en línea mediante alguno de los servidores que tienen habilitada la herramienta mencionada. Al terminar el webinario, las preguntas pendientes al grupo se pueden contestar en una extensión de la sesión o en sesiones adicionales programadas para tal fin; en estas se puede experimentar de forma colaborativa las asesorías entre participantes y tutores o solo entre participantes, habiendo la posibilidad de que surjan nuevos líderes en cada curso.

Desde que se hicieran populares en el año 2009 gracias al desarrollo de aplicaciones que permitieron colocarlos en servidores públicos y privados en línea, se han escrito muchos artículos acerca de los webinarios y su utilización en el mercado, reuniones de trabajo, investigación colaborativa y aplicaciones en educación. Su beneficio va aso- 
ciado a la comodidad de los participantes, especialmente en el ámbito universitario, donde los expertos son cada vez más reacios a viajar y esta herramienta les permite hacer una clase magistral desde su propia aula en un punto fijo, pero con la posibilidad de que la trasmisión llegue a cualquier lugar. Como afirma Quintero (2014), las herramientas son interoperables: puede haber una conferencia en la universidad y los estudiantes la siguen desde su casa.

En el webinario que realizaron los estudiantes se sugirió la utilización de las opciones que herramienta les facilitaba, como por ejemplo compartir escritorio en vivo, a través de Internet, muchos participantes en la sesión, cambiar de orador en cualquier punto de la sesión, facilitar el control remoto de teclado y del mouse a un participante, según el perfil definido, como también planificar de manera antelada las actividades y cumplir con la agenda y los puntos establecidos para tratarse en seminario.

Se considera que las sesiones deben ser enviadas rápidamente a los participantes invitados y a los tutores mediante grabación; se debe utilizar la pizarra multiusuario, hacer uso de chat multiusuario y enviar mensajes a todos o a un grupo identificado de participantes. También es necesario utilizar la transferencia de archivos, además seleccionar alguna aplicación útil para el expositor.

\section{EL WEBINARO COMO HERRAMIENTA DE INTEGRACIÓN EN CLASES VIRTUALES}

La educación virtual exige el uso de metodologías que permitan lograr el nivel de calidad no solo de los contenidos sino también del acceso a los recursos, su disponibilidad y su nivel pedagógico (Campo et al., 2011). La proliferación masiva de sitios web y la diversidad de contenidos para educación formal y no formal tienen a los diseñadores hurgando entre formatos y herramientas para hacer usables los sitios. La web tiene muchos sitios que integran una diversidad de elementos atractivos y cuyos efectos sobre los internautas deben ser medidos (Chardonneau, 2014). Con algunas herramientas en línea, como las definidas por Google (Google Analytics, Universal
Analytics y Google Tag Manager), la utilización de los webinarios también depende del grado de aceptación que se logre en los posibles participantes, además del tema a ser tratado en el mismo. En el mundo de la web, la creación de una estrategia eficaz requerirá el uso de muchos canales interdependientes (Eouzan, 2013).

Según Catasús et al. (2014), las posibilidades que ofrecen las tecnologías y la necesidad de actualizarse hacen que la docencia desarrollada en línea posibilite un proceso colaborativo entre docentes, lo que facilita la mejora de la enseńanza, la formación continua y el desarrollo profesional de quienes la realiza. No solo es el movimiento tecnológico que impulsa a la educación a utilizar sus herramientas, también las nuevas necesidades frente a escenarios distintos los que motivan a la utilización de otras técnicas y herramientas. Como explica Pérez (2011):

La adopción del sistema de créditos ECTS y el nuevo aspecto promovido por la legislación universitaria de la formación en competencias impulsan un cambio en las metodologías docentes hacia métodos de aprendizaje más activo del estudiante en un contexto que se extiende a lo largo de la vida (lifelong learning). De igual manera, la nueva distribución de la docencia en clases magistrales, seminarios y tutorías, establecida en la ordenación docente para los nuevos Grados, permite planificar y programar las distintas materias desde una perspectiva totalmente nueva en la que, junto a los métodos tradicionales, adquieren mayor protagonismo otras técnicas y actividades más innovadoras e interactivas enmarcadas en las clases de Seminario. (p. 256)

Además, las obligaciones que tienen los educadores frente a la sociedad les presentan el reto de llevar al ciudadano una educación de calidad, algo nunca antes planteado y que ahora es posible gracias a todas las herramientas disponibles, aumentando las posibilidades de éxito. Estos puntos son tratados por Souto (2010) dentro del proyecto el derecho fundamental a la educación: derecho, ciudadanía y libertad.

La tendencia más clara en la evolución en el uso de la red es el Internet de cualquier cosa (IoE 
Internet of Everything, por sus siglas en ingles). Importantes fabricantes de equipos para Internet, como Cisco, apuestan a ese contexto, el cual está basado en la colaboración, como lo señala Nof (2015) al explicar que la colaboración en organizaciones altamente distribuidas compuestas por personas, robots y sistemas autónomos debe ser reestructurada debido al aumento de la alta ingeniería. El objetivo es aumentar las habilidades del ser humano en el trabajo a través de esa restructuración, buscando mejorar las condiciones de las organizaciones para que estas logren su misión. La premisa fundamental es que sin un efectivo e-Work y e-Services, el potencial de actividades emergentes como la educación virtual asistida por TIC, e-Commerce, virtual manufacturing, tele-robotic medicine, automated construction, smart energy grid, cyber-supported agriculture, and intelligent transportation no se pueden materializar en forma completa.

El compromiso de la educación para lograr esa transformación debe ser total y las herramientas utilizadas tan novedosas como las que se espera que desarrollen los estudiantes. En este escenario, para el webinario es fundamental mantener a esos estudiantes motivados y atentos para conseguir el mayor provecho de esas novedosas herramientas.

Respecto a las herramientas para captar la atención se instó a los participantes a utilizar el ambiente 3D, haciendo atractiva su presentación. Esta ayuda visual permite utilizar elementos muy coloridos de fácil uso y visualización en la web (Dirksen, 2015). Además, la utilización de WebGL y la integración con los navegadores genera facilidad de usabilidad (Parisi, 2012). Es preciso destacar que el software más utilizado para el desarrollo de la gamificacion de la educación en 2D y 3D es Unity (Horachek, 2014).

\section{Materiales y Métodos}

\section{Tipo y Diseño}

En esta investigación se va a utilizar un enfoque mixto. En lo cuantitativo se analizó cuestionarios y en lo cualitativo se utilizaron entrevistas a informantes; la información de esta últimas se categorizaron y triangularon.
La triangulación se refiere al uso de varios métodos (tanto cuantitativos como cualitativos), de fuentes de datos, de teorías, de investigadores o de ambientes en el estudio de un fenómeno. El término triangulación es tomado de su uso en la medición de distancias horizontales durante la elaboración de mapas de terrenos o levantamiento topográfico, se puede tener una orientación con respecto a los otros dos puntos y localizarse en la intersección (Okuda, 2005). La triangulación diferentes métodos busca analizar un mismo fenómeno a través de diversos acercamientos. Aunque generalmente se utilizan distintas técnicas cualitativas, se puede utilizar técnicas cuantitativas como cualitativas en conjunto. El arte de la triangulación consiste en dilucidar las diferentes partes complementarias de la totalidad del fenómeno y analizar por qué los distintos métodos arrojan diferentes resultados.

En relación al análisis de los registros, los datos cualitativos se triangularon. Las ideas resultantes en cada dimensión reflejaran las observaciones y experiencias de los participantes. Los datos cuantitativos se tradujeron a la inferencia estadística paramétrica, tras de evaluar que la variable aleatoria objeto de estudio sigue una distribución normal (Friendly, 2015). Esta forma de trabajo permitió a los investigadores hacer uso de todas las herramientas disponibles para analizar las dimensiones del tema en cuestión y tener un amplio espectro acerca del uso de los seminarios Web o Webinarios en la educación, específicamente en la universitaria. Cabe preguntarse entonces: ¿será ideal utilizar esa herramienta para los participantes?

\section{Participantes}

Durante los años 2013, 2014 y el primer semestre de 2015 se registraron los datos de los participantes de los cursos de pregrado y postgrado en los programas de Ingeniería Informática en la Universidad Politécnica de la Victoria (UPTA), con Especialización en Gerencia educativa, Especialización en Educación, Especialización en Planificación y evaluación de la educación; Maestría en ciencias de la educación en la Universidad Santa María (USM) y Maestría en gerencia de tecno- 
logías de información y comunicación, Maestría en tecnología educativa, Maestría en gerencia de recursos humanos en la Universidad Nacional Experimental de la Fuerzas Armadas (UNEFA); también se registró su relación con el webinario como parte de su sistema de aprendizaje personal. Las tres universidades pertenecen a la zona central de Venezuela, el estado Aragua. La UPTA está adscrita al ministerio para la educación universitaria; la UNEFA, al ministerio para la defensa y la USM, a entes privados.

Los participantes estaban inscritos en los cursos impartidos por los autores de esta investigación, distribuidos de la siguiente forma:

- UPTA: Introducción a la simulación por eventos discretos (pregrado), Redes avanzadas (pregrado), Sistemas Operativos (pregrado);

- USM: Diseño y evaluación de redes participativas y colaborativas (postgrado), Planificación estratégica para la educación (postgrado), Tecnología de la Información y sus Aplicaciones al Campo Educativo (postgrado), Planificación estratégica en educación (postgrado) y

- UNEFA: Sistemas de información aplicados a la gerencia de recursos humanos (postgrado), Gerencia de proyectos y las TIC (postgrado), Auditoria de sistemas (postgrado).

Los participantes eligieron libremente las herramientas para la realización de los webinarios, dentro de la lista de opciones siguiente: Webex, Anymeeting, Mikogo y Openmeetings.

El método de muestreo seleccionado fue el casual o incidental no probabilístico: Se trató de un proceso en el que los investigadores seleccionaron directa e intencionalmente a los individuos de la población. Fueron los profesores universitarios quienes registraron a los alumnos cuyas opiniones permitieron evaluar a los seminarios virtuales en la web o webinarios. La muestra quedó conformada por 864 individuos de tres universidades, en dos áreas (tecnología y educación), encuestados durante 2 ańos y medio, en 10 cursos.

\section{Instrumento}

El enfoque cuantitativo del estudio se realizó con la medición de las variables con escala numérica. Estos datos se analizaron utilizando la estadística descriptiva e inferencial, sin manipulación de variables, estas se midieron tal como se registraron de los participantes. Para recabar datos cualitativos de algunos participantes, los autores realizaron algunas entrevistas a informantes claves. La información obtenida se trianguló con la finalidad de develar sensaciones y expectativas.

Para la recolección de los datos cuantitativos se utilizó un cuestionario aplicado por Peña (2012) en una investigación similar que analizó el uso de los mundos virtuales. El instrumento se denomina Análisis de vivencias y experiencias educativas realizadas en los webinarios. Presenta opciones de respuesta politómicas, con escalamiento tipo Likert. Se utilizó el sistema de puntuación simple.

Esta encuesta consta de tres dimensiones, la primera dimensión consta de preguntas orientadas a la recolección de información personal, tal como edad, sexo, programa académico.

La segunda dimensión consta de respuestas: (1) Muy difícil, (2) Difícil, (3) Fácil, (4) Muy fácil y (5) No sabe/no contesta. Con los ítems de esta dimensión se busca conocer el impacto que tuvo para el participante al enfrentarse inicialmente a la herramienta utilizada para el webinario, sus accesorios de software y sus recursos.

La tercera dimensión ofreció como opciones de respuestas: (1) Nada, (2) Poco, (3) Bastante, (4) Mucho y (5) No sabe/no contesta. Con esta dimensión se pretendió conocer las apreciaciones de los participantes de los cursos en relación con el uso académico del webinario en su contexto educativo.

El instrumento es muy utilizado en el mundo entero en la investigación educativa y se basa en una serie de preguntas o ítems dirigidos a los sujetos con la finalidad de describir el problema objetivo de la indagación (Del Rincón, Arnal, Latorre y Sans, 1995). La finalidad del cuestionario es obtener de manera sistemática y ordenada, de la población investigada información acerca de las variables de interés. En la investigación cualita- 
tiva, esta información está asociada a las ideas y reflexiones de los informantes relacionadas con el sustento teórico del sistema analizado (Marradi, 2012).

La validez del cuestionario. En cualquier tipo de investigación, la capacidad del instrumento para la recogida rigurosa de los datos depende de dos atributos importantes: la validez y la fiabilidad. La validez se relaciona con la utilidad del instrumento, es decir, verifica que este mida aquello que se pretende medir (Fox, 1981). En este caso se ha aplicado una validez de contenido mediante el juicio de expertos, por ser la técnica más potente que puede usar el investigador.

Para esta investigación, el cuestionario fue validado por juicio de expertos, estableciendo así su validez de contenido. Una vez recogidas todas las objeciones realizadas por los jueces, se realizó una depuración general de errores y omisiones en los campos de cada uno de los registros, hasta llegar a su formalización definitiva.

La confiabilidad del cuestionario. La fiabilidad de un cuestionario determina la consistencia interna de los resultados obtenidos por los mismos individuos cuando son cumplimentados en diferentes ocasiones. La fiabilidad es una medida del grado de consistencia o concordancia que puede expresarse mediante algún coeficiente de correlación. Al ser la fiabilidad una medida del grado de consistencia o concordancia puede expresarse mediante algún coeficiente de correlación (Pérez, 2011), en la Teoría Clásica de los Tests (TCT) la verificación de la consistencia interna permite estimar la fiabilidad de pruebas, escalas o test cuando los ítems o reactivos utilizados miden el mismo atributo. Según Ledesma, Molina y Valero (2002), dentro de la Teoría Clásica de los Tests (TCT) el método de consistencia interna es el camino más habitual para estimar la fiabilidad de pruebas, escalas o test, cuando se utilizan conjuntos de ítems o reactivos que se espera midan el mismo atributo o campo de contenido. La principal ventaja de ese método es que requiere sólo una administración de la prueba; además, los principales coeficientes de estimación basados en este enfoque son sencillos de computar y están disponibles como opción de análisis en los programas estadísticos más co- nocidos, como SPSS, Statistic o SAS, Excel. (p. 143)

Se usó el coeficiente de confiabilidad Alfa de Cronbach, que es, sin duda, el más utilizado por los investigadores para verificar la consistencia interna de los cuestionarios. Este coeficiente puede tomar valores entre 0 y 1 , donde 0 significa fiabilidad nula y 1 representa fiabilidad total. Cuanto mayor sean las correlaciones lineales entre ítems, mayor será el valor alfa (Peña y Ortega, 2012).

Los resultados del coeficiente Alfa de Cronbach según dimensión indicaron:

- 0,93 (Confiabilidad elevada) para la dimensión I (ítem 1.1 - 1.4)

- 0,96 (Confiabilidad elevada) para la dimensión II (ítem 2.1 - 2.7)

- 0,86 (Confiabilidad aceptable) para la dimensión III (ítem 3.1 - 3.27)

El análisis estadístico se realizó usando un software libre, pero que posea un soporte estadístico sólido con un nivel superior al promedio de usabilidad. El software utilizado fue el "Statics Open For All", que se puede conectar a diferentes bases de datos y los resultantes se pudieran exportar a diferentes formatos. En esta investigación se trabajó con SQLite.

Los servidores de Internet utilizados para los webinarios fueron 4: Anymeeting, Openmeetings, Webex y Mikogo. Aunque existe una gran variedad de opciones, estos fueron los más seleccionados por los participantes. Las versiones para demostración fueron suficientes para el trabajo realizado y, en algunos casos donde se disponía de poco número de conexiones, los participantes se ubicaban en una locación y el profesor en otra, al igual que el expositor.

Las herramientas utilizadas en la investigación por los equipos participantes se encuentran ubicadas en los siguientes sitios:

- Openmeetings, producto de código abierto para presentaciones, conferencias web, pizarra de dibujo, colaboración y edición de documentos, intercambio de escritorio del usuario, basado en RIA de OpenLaszlo y el servidor de video Red5, y MyS- 
QL como base de datos más utilizada. (http://openmeetings.apache.org/)

- AnyMeeting, que permite crear webinar o reuniones online de manera gratuita para generar un espacio virtual en la web. (http://www.anymeeting.com/en/)

- WebEx, cuya licencia es propiedad de Cisco; tiene una versión gratuita para 2 conexiones más el expositor. (https://www.webex.com/)

- Mikogo, multiplataforma que admite hasta 25 participantes, con posibilidad de compartir el escritorio, el contenido de la pantalla, realizar conferencia web o aplicación por Internet. (https://www.mikogo.com/download/)

\section{Procedimiento}

Las sesiones del webinario necesitaban de un proceso previo a su realización y otro durante el desarrollo del mismo; esto para los cursos de cada universidad involucrada. En primer lugar era indispensable que los organizadores del webinario (máximo 3 estudiantes) hicieran una invitación al grupo del curso respectivo, detallando la forma de acceso al servidor seleccionado y los software necesarios para la participación fluida, además de promocionar el tema y al expositor.

Para el éxito del webinario fue necesario definir algunas características:

1. Entender las perspectivas de los participantes y el expositor, quienes pueden estar en ubicaciones diferentes y con elementos distractores muy fuertes; pueden utilizar herramientas para captar la atención con la imagen, los sonidos, la solicitud de opiniones o las recompensas por participación entre otros. La duración recomendada fue de 45 minutos de máximo aprovechamiento e interactividad virtual.

2. Los webinarios permiten incorporar a más personas en un área geográfica más amplia, con costos razonables y sin la dificultad de la organización de reuniones presenciales.

3. La interacción visual es importante, pero al compartir el escritorio se pierde y solo queda la voz como elemento de captura de la aten- ción junto con las aplicaciones, se debe poner mucha atención en este aspecto.

4. Dominar la herramienta a utilizar para el webinario, es una mala práctica perder minutos en ajustes por falta de experticia en la plataforma, planteamiento similar de Area (2014).

Cada uno de los equipos de trabajo que ejecutan los webinarios en cada curso están integrados por un expositor del tema, además de un segundo integrante como el encargado de la logística y soporte técnico antes y durante la sesión; el tercer integrante del equipo, como moderador y gestor del debate, responde las preguntas generales de los participantes por medio del chat de texto y cede el turno en la ronda de intervenciones.

En la sección de resultados se presenta el testimonio de la informante 1 perteneciente a la UPTA, una líder natural generada en las primeras sesiones de clase. Con su testimonio se logró develar ideas sobre la relación entre los participantes y el webinario. Adicionalmente, en la tabla 8 , se presenta la triangulación compuesta por cuatro columnas: categoría, postulado teórico, informante, postura del investigador. Luego, en la figura 1, se resume la triangulación con los puntos clave. 


\section{Resultados}

Luego de registrar los datos (cuantitativos y cualitativos) se obtuvieron los grupos que se muestran en la tabla 1 clasificados según sexo, edad, programa al cual pertenecen y la universidad en la cual están inscritos los participantes.

Tabla 1. Distribución registrada de las muestras

\begin{tabular}{llcc}
\hline Edad & Ítem & \multicolumn{1}{c}{$f$} & $\%$ \\
\hline Edad & (1) Menos de 25 & 440 & 50.9 \\
& (2) $25-30$ & 376 & 43.5 \\
& (3) 30 - 35 & 33 & 3.8 \\
& (4) Más de 35 & 15 & 1.7 \\
\hline Programa & (1) Tecnología & 661 & 76.5 \\
& (2) Educación & 203 & 23.5 \\
\hline Sexo & (1) Mujer & 455 & 52.7 \\
& (2) Hombre & 409 & 47.3 \\
\hline Universidad & (1) UPTA & 453 & 52.4 \\
& (2) USM & 197 & 22.8 \\
& (3) UNEFA & 214 & 24.8 \\
\hline
\end{tabular}

La tabla 2 muestra los valores registrados para cada uno de los ítems de la segunda parte del cuestionario donde se buscó conocer cómo fue el primer impacto de los estudiantes frente el uso de la herramienta webinario.

Tabla 2. Segunda parte del cuestionario: Primer impacto del participante en la herramienta
La tercera parte del cuestionario (tabla 3 en la página siguiente), contempla los ítems que registran la percepción de los participantes ante su vida académica y la percepción de su progreso académico al igual que su motivación, luego del uso del webinario. Un punto de interés es cómo esa herramienta relaciona a los participantes y los tutores y si estos últimos están capacitados para enfrentar ese nuevo escenario académico.

\begin{tabular}{llrrrrr}
\hline \multicolumn{1}{c}{ Ítem } & & $\begin{array}{c}\text { Muy } \\
\text { difícil }\end{array}$ & Difícil & Fácil & $\begin{array}{r}\text { Muy } \\
\text { fácil }\end{array}$ & $\begin{array}{c}\text { No sabel } \\
\text { No contesta }\end{array}$ \\
\hline 2.1 Primer acceso & $f$ & 56 & 430 & 201 & 174 & 3 \\
& $\%$ & 6.5 & 49.8 & 23.3 & 20.1 & 0.3 \\
\hline 2.2. El manejo de las & $f$ & 92 & 436 & 191 & 143 & 2 \\
herramientas de movilidad & $\%$ & 10.6 & 50.5 & 22.1 & 16.6 & 0.2 \\
\hline 2.3. El acceso al chat de texto & $f$ & 36 & 100 & 591 & 133 & 4 \\
& $\%$ & 4.2 & 11.6 & 68.4 & 15.4 & 0.5 \\
\hline 2.4. El comunicarte con los & $f$ & 6 & 136 & 196 & 596 & 0 \\
amigos a través del chat & $\%$ & 0.7 & 15.7 & 22.7 & 60.9 & 0 \\
\hline 2.5. La comunicación a través & $f$ & 0 & 123 & 510 & 231 & 0 \\
del Chat de voz & $\%$ & 0 & 14.2 & 59.0 & 26.7 & 0 \\
\hline 2.6. El acceder o salir de un & $f$ & 11 & 443 & 267 & 134 & 9 \\
grupo & $\%$ & 1.3 & 51.3 & 30.9 & 15.5 & 1.0 \\
\hline 2.7. Hacer fotos y guardarlas & $f$ & 53 & 325 & 234 & 252 & 0 \\
& $\%$ & 6.1 & 37.6 & 27.1 & 29.2 & 0 \\
\hline
\end{tabular}


Tabla 3. Tercera parte del cuestionario: Impacto de la utilización del webinario en el contexto educativo del participante

\begin{tabular}{|c|c|c|c|c|c|c|}
\hline Ítem & & Nada & Poco & Bastante & Mucho & $\begin{array}{l}\text { No sabel } \\
\text { No contesta }\end{array}$ \\
\hline \multirow[t]{2}{*}{ 3.1. Las tutorías personales fueron de utilidad } & $f$ & 9 & 130 & 209 & 516 & 0 \\
\hline & $\%$ & 1.0 & 15.0 & 24.2 & 59.7 & 0 \\
\hline \multirow{2}{*}{$\begin{array}{l}\text { 3.2. Superaste las dificultades de acceso y uso gracias a } \\
\text { tus tutores }\end{array}$} & $f$ & 56 & 430 & 201 & 174 & 3 \\
\hline & $\%$ & 6.5 & 49.8 & 23.3 & 20.1 & 0.3 \\
\hline \multirow{2}{*}{$\begin{array}{l}\text { 3.3. Superaste las dificultades de acceso y uso gracias a } \\
\text { colegas }\end{array}$} & $f$ & 92 & 436 & 191 & 143 & 2 \\
\hline & $\%$ & 10.6 & 50.5 & 22.1 & 16.6 & 0.2 \\
\hline \multirow{2}{*}{$\begin{array}{l}\text { 3.4. Superaste las dificultades de acceso y uso gracias a } \\
\text { tutoriales }\end{array}$} & $f$ & 36 & 100 & 591 & 133 & 4 \\
\hline & $\%$ & 4.2 & 11.6 & 68.4 & 15.4 & 0.5 \\
\hline \multirow{2}{*}{$\begin{array}{l}\text { 3.5. Superaste las dificultades de acceso y uso por inves- } \\
\text { tigación }\end{array}$} & $f$ & 6 & 136 & 196 & 526 & 0 \\
\hline & $\%$ & 0.7 & 15.7 & 22.7 & 60.9 & 0 \\
\hline \multirow{2}{*}{$\begin{array}{l}\text { 3.6. Ha modificado los hábitos de organización de tu tiempo } \\
\text { libre }\end{array}$} & $f$ & 0 & 123 & 510 & 231 & 0 \\
\hline & $\%$ & 0 & 14.2 & 59.0 & 26.7 & 0 \\
\hline \multirow[t]{2}{*}{ 3.7. La interacción ha modificado tus hábitos de sueño } & $f$ & 11 & 443 & 267 & 134 & 0 \\
\hline & $\%$ & 1.3 & 51.3 & 30.9 & 15.5 & 0 \\
\hline \multirow[t]{2}{*}{ 3.8. Te motivaría a realizar actividades académicas } & $f$ & 53 & 325 & 234 & 252 & 0 \\
\hline & $\%$ & 6.1 & 37.6 & 27.1 & 29.2 & 0 \\
\hline \multirow[t]{2}{*}{ 3.9. Te ha permitido relacionarte mejor que en la vida real } & $f$ & 373 & 224 & 144 & 32 & 91 \\
\hline & $\%$ & 43.2 & 25.9 & 16.7 & 3.7 & 10.5 \\
\hline \multirow[t]{2}{*}{ 3.10. Más libre de expresar tus ideas que en la vida real } & $f$ & 193 & 431 & 198 & 23 & 19 \\
\hline & $\%$ & 22.3 & 49.9 & 22.9 & 2.7 & 2.2 \\
\hline \multirow[t]{2}{*}{ 3.11. Te ha motivado a aprender en el webinario } & $f$ & 66 & 45 & 556 & 168 & 29 \\
\hline & $\%$ & 7.6 & 5.2 & 64.4 & 19.4 & 3.4 \\
\hline \multirow{2}{*}{$\begin{array}{l}\text { 3.12. Asistir a clase dentro del webinario te resultó de } \\
\text { interés }\end{array}$} & $f$ & 42 & 164 & 475 & 183 & 0 \\
\hline & $\%$ & 4.9 & 19.0 & 55.0 & 21.2 & 0 \\
\hline \multirow{2}{*}{$\begin{array}{l}\text { 3.13. Visualizar presentaciones multimedia te resultó de } \\
\text { interés }\end{array}$} & $f$ & 136 & 187 & 433 & 79 & 29 \\
\hline & $\%$ & 15.7 & 21.6 & 50.1 & 9.1 & 3.4 \\
\hline \multirow[t]{2}{*}{ 3.14. Asistir a tutorías te resultó de interés } & $f$ & 128 & 185 & 419 & 77 & 55 \\
\hline & $\%$ & 14.8 & 21.4 & 48.5 & 8.9 & 6.4 \\
\hline \multirow[t]{2}{*}{ 3.15. Sesiones colectivas de aclaración te resultó de interés } & $f$ & 58 & 380 & 243 & 80 & 93 \\
\hline & $\%$ & 7.9 & 44.0 & 28.1 & 9.3 & 10.8 \\
\hline \multirow[t]{2}{*}{ 3.16 Realizar encuentros amistosos fue interés } & $f$ & 0 & 129 & 253 & 482 & 0 \\
\hline & $\%$ & 0 & 14.9 & 29.3 & 55.8 & 0 \\
\hline \multirow[t]{2}{*}{ 3.17. Resolver problemas colaborativamente fue de interés } & $f$ & 19 & 129 & 240 & 435 & 41 \\
\hline & $\%$ & 2.2 & 14.9 & 27.8 & 50.3 & 4.7 \\
\hline \multirow[t]{2}{*}{ 3.18. Intercambiar ideas entre colegas } & $f$ & 56 & 431 & 201 & 173 & 3 \\
\hline & $\%$ & 6.5 & 49.9 & 23.3 & 20.0 & 0.3 \\
\hline \multirow[t]{2}{*}{ 3.19. Poner en común inquietudes } & $f$ & 92 & 436 & 191 & 143 & 2 \\
\hline & $\%$ & 10.6 & 50.5 & 22.1 & 16.6 & 0.2 \\
\hline \multirow{2}{*}{ 3.20. Mejoró tu capacidad para comunicarte digitalmente } & $f$ & 36 & 100 & 591 & 133 & 4 \\
\hline & $\%$ & 4.2 & 11.6 & 68.4 & 15.4 & 0.5 \\
\hline 3.21. Comunicarte con voz te resultó de interés & $f$ & 6 & 136 & 197 & 525 & 0 \\
\hline & $\%$ & 0.7 & 15.7 & 22.8 & 60.8 & 0 \\
\hline 3.22. Comunicarte a través del chat te resultó de interés & $f$ & 0 & 124 & 509 & 231 & 0 \\
\hline & $\%$ & 0 & 14.4 & 58.9 & 26.7 & 0 \\
\hline 3.23. Comunicación multimedia es importante en los & $f$ & 11 & 442 & 268 & 134 & 9 \\
\hline aprendizaje & $\%$ & 1.3 & 51.2 & 31.0 & 15.5 & 1.0 \\
\hline 3.24. La comunicación visual utilizada fue motivadora & $f$ & 53 & 325 & 234 & 252 & 0 \\
\hline & $\%$ & 6.1 & 37.6 & 27.1 & 29.2 & 0 \\
\hline 3.25. Tutores utilizaron metodologías adecuadas & $f$ & 372 & 225 & 144 & 32 & 91 \\
\hline & & 43.1 & 26.0 & 16.7 & 3.7 & 10.5 \\
\hline
\end{tabular}


En la tabla 6 se muestra la medida de normalidad de las curvas que representan los registros de los datos capturados. Se observa algunos de los resultados de varios ítems y los valores de los parámetros sesgo y curtosis.

Tabla 6. Valores de Sesgo y Curtosis para algunos de los parámetros registrados

\begin{tabular}{|c|c|c|}
\hline Ítem & Sesgo & Curtosis \\
\hline 2.1. El primer acceso al webinario & 0.405 & -0.788 \\
\hline $\begin{array}{l}\text { 2.5. La comunicación a través del Chat de } \\
\text { voz }\end{array}$ & -0.1 & -0.522 \\
\hline 2.6. El acceder o salir de un grupo & 0.712 & -0.372 \\
\hline $\begin{array}{l}\text { 3.4. Superaste las dificultades de acceso y } \\
\text { uso de webinario gracias a tus tutores }\end{array}$ & 0.405 & -0.788 \\
\hline $\begin{array}{l}\text { 3.4. Superaste las dificultades de acceso } \\
\text { y uso de webinario gracias a la ayuda de } \\
\text { colegas }\end{array}$ & 0.435 & -0.594 \\
\hline $\begin{array}{l}\text { 3.6. La interacción en webinario ha modi- } \\
\text { ficado los hábitos de organización de tu } \\
\text { tiempo libre }\end{array}$ & -0.1 & -0.522 \\
\hline $\begin{array}{l}\text { 3.7. La interacción en webinario ha modifi- } \\
\text { cado tus hábitos de sueño }\end{array}$ & 0.712 & -0.372 \\
\hline $\begin{array}{l}\text { 3.12. Asistir a clase dentro del webinario te } \\
\text { resultó de interés }\end{array}$ & -0.514 & 0.125 \\
\hline $\begin{array}{l}\text { 3.13. Visualizar presentaciones multimedia } \\
\text { en nuestro espacio virtual te resultó de } \\
\text { interés }\end{array}$ & -0.023 & -0.086 \\
\hline $\begin{array}{l}\text { 3.14. Asistir a tutorías virtuales personali- } \\
\text { zadas en nuestro espacio virtual dentro del } \\
\text { webinario te resultó de interés }\end{array}$ & 0.148 & -0.064 \\
\hline $\begin{array}{l}\text { 3.16. Realizar encuentros amistosos dentro } \\
\text { del webinario te resultó de interés }\end{array}$ & -0.81 & -0.724 \\
\hline $\begin{array}{l}\text { 3.17. Resolver problemas colaborativamen- } \\
\text { te dentro del webinario te resultó de interés }\end{array}$ & -0.656 & -0.114 \\
\hline $\begin{array}{l}\text { 3.18. Intercambiar ideas entre colegas } \\
\text { dentro de webinario te ayudó a fortalecer } \\
\text { las competencias tecnológicas }\end{array}$ & 0.409 & -0.78 \\
\hline $\begin{array}{l}\text { 3.19. Poner en común inquietudes en } \\
\text { webinario te resultó de interés }\end{array}$ & 0.435 & -0.594 \\
\hline $\begin{array}{l}\text { 3.22. Comunicarte a través del chat en el } \\
\text { webinario te resultó de interés }\end{array}$ & -0.101 & -0.529 \\
\hline $\begin{array}{l}\text { 3.23. El buen uso de las herramientas de } \\
\text { comunicación multimedia desempeña un } \\
\text { papel importante en los procesos de apren- } \\
\text { dizaje en webinarios }\end{array}$ & 0.709 & -0.374 \\
\hline $\begin{array}{l}\text { 3.24. La comunicación visual utilizada en } \\
\text { webinario durante el curso fue motivadora }\end{array}$ & -0.031 & -1.149 \\
\hline $\begin{array}{l}\text { 3.25. Los tutores utilizaron metodologías } \\
\text { adecuadas para fomentar la actividad } \\
\text { creativa del webinario }\end{array}$ & 1.031 & -0.004 \\
\hline
\end{tabular}

Para el análisis cualitativo de la situación planteada se desarrolló un grupo de entrevistas y vivencias de los tutores para reconocer los sentimientos y conflictos emocionales presentes en los escenarios educativos asistidos por webinarios. En este sentido, se presenta el relato de una de las participantes en el cual se nota una particular integración con el proceso.
El siguiente de la informante 1 permitió conformar un mapa de criterios relacionados con las frases importantes encontradas en ese relato. Esta información se podría considerar en la futura implementación de esta herramienta virtual en un programa académico formal en una universidad venezolana.

\section{Testimonio}

El Webinario permite una actividad académica sincrónica en la que participa el estudiante (tanto presencial como virtualmente), siendo posible evaluar los contenidos cognitivos, procedimentales y sobre todo actitudinales del curso. Estos son muy importantes para evaluar el proceso educativo del estudiante en el mundo real. Se pensó en las posibilidades de utilizar webinario para el desarrollo de distintos cursos, como apoyo a las clases presenciales para dar formación a alumnos $e$ incluso crear grupos de apoyo. Con los webinarios se fomenta el trabajo colaborativo y el compañerismo; de esta forma un grupo reducido de alumnos creamos sesiones de trabajo muy productivas.

Logramos superar las dudas iniciales. A medida que avanzó la experiencia destaqué como lider por encima del resto, tanto en la mayor caracterización de mi participación como por mis prontos conocimientos para realizar las tareas, asi como para resolver las dudas del resto de compañeros.

Quedábamos en reunirnos a determinadas horas para aclarar dudas $y$, sin darme cuenta, me convertí en un facilitador. Por tanto, creé una dinámica de trabajo diferente y fuera de la organización inicial que los tutores habian establecido. Es curioso cómo la buena imagen que di en los webinarios, tanto fisica como virtualmente, hizo que todos tuvieran una percepción muy favorable sobre mi. En sucesivas sesiones presenciales fui aceptada con mucho agrado tanto por alumnos como por profesores. Como producto de mi condición de lider, me sentí observada desde cerca por el grupo de tutores.

Alprincipio, la comunicación que utilizamos fue el chat escrito general, hasta que descubrimos que existen chats privados entre pares de participantes y un chat de voz que nos dio muchas dificulta- 
des. Creamos un grupo de chat de voz solo para miembros del mismo; aun así, se generaba problemas de conectividad y ruido. Los controles de sonido me permitieron regular los micrófonos de mis compañeros y reducir el uso de los datos en la red dejando solo el sonido de voz y graduando el volumen al expositor del momento.

El principal beneficio es que el estudiante se siente capaz de realizar una labor académica en un medio virtual y percibe que él es centro del proceso educativo. Como consecuencia de ello se responsabiliza de su propio aprendizaje, mediante una motivación previa por las herramientas tecnológicas.

El entorno virtual obliga al profesor a reflexionar sobre el contenido de su curso, a dosificar mejor los contenidos del mismo; los grupos de apoyo o asesoría funcionan muy. En nuestro caso, el webinario era parte de nuestra evaluación de desempeño en el curso y debiamos hacer unas actividades de planificación, motivación y promoción, lo que generaba un nivel de estrés adicional al ya experimentado en los seminarios tradicionales. En el webinario, el estudiante sabe que no sólo es un estudiante sino que además pertenece a una comunidad, se siente perteneciente a un grupo. No sólo entra a clase, sino que también se socializa. Este es un entorno donde también se puede enseñar, lo que hace que el participantes se sienta capaz.

A continuación se transcriben las frases claves generadas en las conversaciones con la informante 1 :

- Creación de grupos de apoyo para personas

- Se logró superar las dudas iniciales

- Rápido progreso de unos respecto a otros

- A medida que avanza la experiencia, destaqué como líder

- Se puede resolver las dudas del resto de compañeros

- Se fomenta el trabajo colaborativo y el compañerismo

- Sin darme cuenta, acabé convirtiéndome en algo similar a un tutor
- Ayudé tanto al tutor real como al director de la investigación a atender las preguntas de mis compañeros

- Creé una dinámica de trabajo diferente y fuera de la organización

- El fenómeno del alumno-líder que se produjo en mi persona fue observado desde cerca por el grupo de tutores.

En la tabla 8 (página siguiente) se presenta una matriz de triangulación entre la teoría del área y la percepción del tutor. Así se contrasta las percepciones sobre este tema. Luego, en la figura 1, se muestra los criterios generados y su interrelación.

En la figura 1 se advierten las distintas categorías que afloraron en las entrevistas a los informantes claves, en especial a la participante 1 que evoluciono como asistente de los tutores.

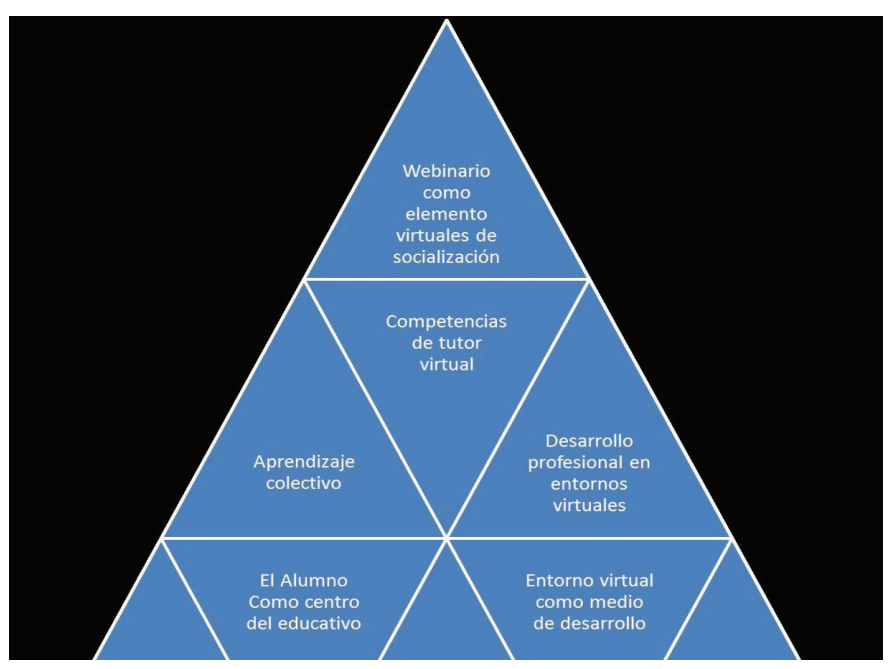

Figura 1. Las categorías presentes en la triangulación, según análisis cualitativo de las entrevistas. Fuente: Peña, Rincón y Tovar.

Luego de organizar y clasificar los datos recopilados, se verificó la distribución normal de los datos y se graficaron las curvas de distribución normal platicúrtica. La figura 2 representa el ítem 3.22 (Interactuar en el webinario mejoró tu capacidad para comunicarte digitalmente). Se observa que muchos de los participantes (68\%) mejoraron su capacidad de comunicarse, una condición básica para los encuentros en el webinario. 
Tabla 8. Matriz de triangulación, postulados teóricos, informant e investigador

\begin{tabular}{|c|c|c|c|}
\hline Categoría & Postulado teórico & Informante & Postura del investigador \\
\hline $\begin{array}{l}\text { Aprendizaje } \\
\text { colaborativo }\end{array}$ & $\begin{array}{l}\text { El aprendizaje colaborativo (AC) } \\
\text { suministra un ambiente que enriquece } \\
\text { el proceso de aprendizaje. Median- } \\
\text { te la introducción de compañeros } \\
\text { interactivos en el sistema educativo } \\
\text { se crean contextos sociales realistas, } \\
\text { con el consiguiente aumento de la } \\
\text { efectividad del sistema. El AC juega } \\
\text { un papel importante en el desarrollo } \\
\text { constructivo del conocimiento, debido } \\
\text { a que la interacción entre estudiantes } \\
\text { es compartida, en contraste con las } \\
\text { interacciones profesor-estudiante, } \\
\text { donde, usualmente, el primero posee } \\
\text { el control sin permitir que siga un pro- } \\
\text { ceso natural de aprendizaje (Cabero, } \\
\text { 2003) }\end{array}$ & $\begin{array}{l}\text { Son más colaboradores y más prestos a } \\
\text { ofrecer apoyo a sus compañeros y eso } \\
\text { favorece el aprendizaje. Se enseña, se } \\
\text { comparte conocimiento, los grupos de } \\
\text { apoyo o asesoría funcionan muy bien } \\
\text { para los alumnos de los webinario }\end{array}$ & $\begin{array}{l}\text { El aprendizaje colaborativo se pone de } \\
\text { manifiesto en todo momento. Partiendo } \\
\text { del ejercicio de integración de los } \\
\text { actores y la puesta en práctica de los } \\
\text { postulados teóricos, se evidencia el alto } \\
\text { nivel de compenetración y compromiso. } \\
\text { Según lo expresado por la informante, } \\
\text { se puede considerar que los webinarios } \\
\text { son espacios donde en esa interacción } \\
\text { social asistida por las TIC, confluyen } \\
\text { en cada participante sus intereses, } \\
\text { experiencias y aprendizajes previos. } \\
\text { El nuevo concepto es muchas veces } \\
\text { transformado, tomando en cuenta las } \\
\text { discusiones con sus pares virtuales y } \\
\text { los tutores. }\end{array}$ \\
\hline $\begin{array}{l}\text { Socialización } \\
\text { en las aulas } \\
\text { virtuales }\end{array}$ & $\begin{array}{l}\text { Con base en los aportes de Esté } \\
\text { (2006), quien plantea los aspectos } \\
\text { pedagógicos de los mundos virtuales } \\
\text { fundamentados en los ejes, a saber: } \\
\text { la interacción constructiva, dada la im- } \\
\text { portancia del juego social como forma } \\
\text { de construir conocimientos, propicia la } \\
\text { creatividad, la duda y la deliberación } \\
\text { y la cohesión comunitaria, en tanto } \\
\text { que reintegración física y ética de las } \\
\text { comunidades como propósito y esen- } \\
\text { cia de la acción educativa, tarea tanto } \\
\text { del estado como de la educación, } \\
\text { generadoras de valores, símbolos y } \\
\text { proyectos. } \\
\text { Caballero (2010) expresa en sus } \\
\text { trabajos sobre el docente que la } \\
\text { identidad en el seminario web y los } \\
\text { escenarios virtuales es un elemento } \\
\text { asociado a la dignidad y el reconoci- } \\
\text { miento por el otro. }\end{array}$ & $\begin{array}{l}\text { Sin darme cuenta acabé convirtiéndome } \\
\text { en un facilitador, en este sentido se } \\
\text { fomenta el trabajo en equipo y permite a } \\
\text { los participantes y profesores interactuar } \\
\text { y colaborar en nuestras actividades. } \\
\text { Distintos grupos se integraron para } \\
\text { desarrollar sesiones extras. }\end{array}$ & \\
\hline
\end{tabular}

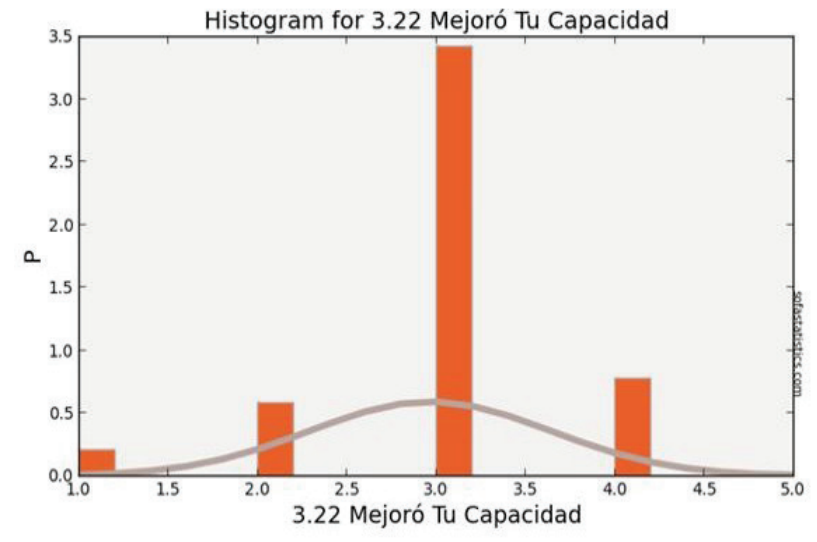

Figura 2. Histograma con resultados del ítem 3.22.

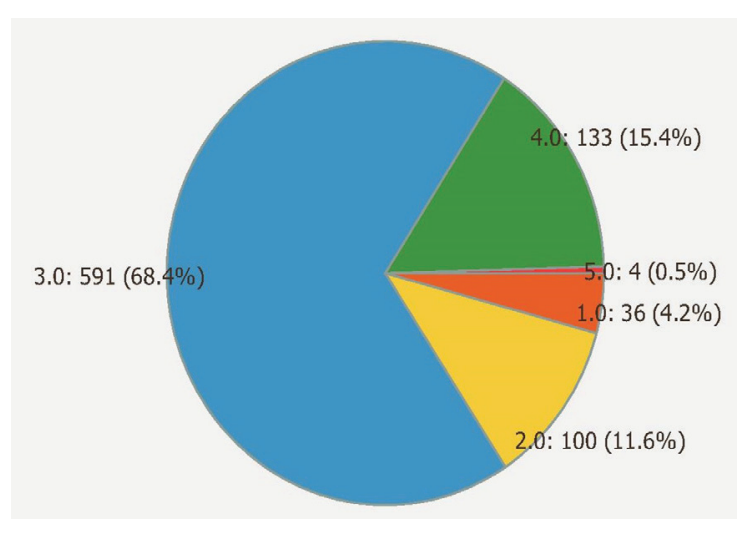

Figura 3. Frecuencia porcentual de resultados para el ítem 3.22: Interactuar en el webinario mejoró tu capacidad para comunicarte digitalmente. 
La figura 4 muestra el nivel de motivación creado en el webinario para realizar actividades académicas. Más del $56 \%$ se vio motivado a realizar actividades académicas; solo poco más del $6 \%$ lo consideró nada motivante. Si se utiliza con mayor frecuencia esta herramienta, se lograría mayor motivación en los participantes.

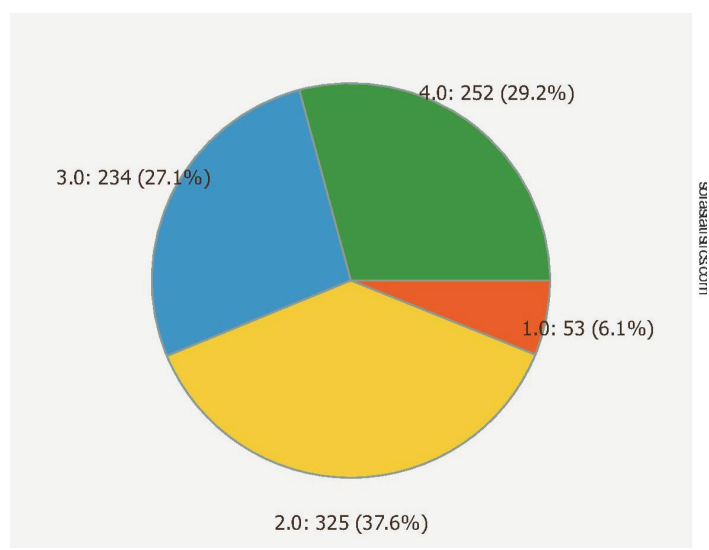

Figura 4. Frecuencia porcentual de resultados para el ítem 3.09: La interacción que se experimenta en webinario te motivaría a realizar actividades académicas.

En la figura 5 se observa que, luego de su experiencia con el webinario, los participantes se motivaron a realizar actividades académicas, pero con un elemento novedoso relacionado con poca dependencia de sus tutores. El resultado indica que comienzan a ser más independientes y adquieren cierto nivel de libertad en el aprendizaje.

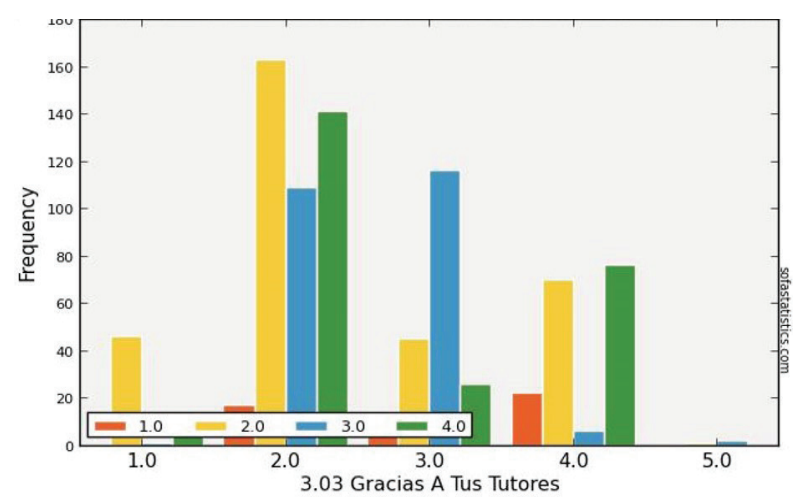

Figura 5. Frecuencia porcentual para los resultados del ítem 3.09: La interacción que se experimenta en webinario te motivaría a realizar actividades académicas en relación con 3.03 Superaste las dificultades de acceso y uso de webinario gracias a tus tutores.

Según la tabla 9 se puede notar que la percepción del primer acceso al webinario fue difícil, tanto en mujeres como en hombres. Esto es algo que pudiera desalentar su utilización, pero, como se verá en los análisis siguientes, esto no fue una limitación para que los estudiantes sacaran el mayor provecho de esta herramienta.

Posteriormente, en la figura 6, se notará que a casi 300 estudiantes que consideraron difícil su primer encuentro con el webinario, la interacción en la plataforma los motivó bastante a aprender según lo expresado con el color azul.

Tabla 9. Precepción del primer acceso al webinario según el Sexo

\begin{tabular}{|c|c|c|c|c|c|c|c|c|c|c|c|c|}
\hline \multirow[b]{2}{*}{ Sexo } & \multicolumn{2}{|c|}{ Muy difícil } & \multicolumn{2}{|c|}{ Difícil } & \multicolumn{2}{|c|}{ Fácil } & \multicolumn{2}{|c|}{ Muy fácil } & \multicolumn{2}{|c|}{$\begin{array}{l}\text { No sabe/ } \\
\text { No con- } \\
\text { testa }\end{array}$} & \multicolumn{2}{|c|}{ Total } \\
\hline & $f$ & $\%$ & $f$ & $\%$ & $f$ & $\%$ & $f$ & $\%$ & $f$ & $\%$ & $f$ & $\%$ \\
\hline Mujer & 36 & 64.3 & 218 & 50.7 & 92. & 45.8 & 108 & 62.1 & 1 & 33.3 & 455 & 52.7 \\
\hline Hombre & 20 & 35.7 & 212 & 49.3 & 109 & 54.2 & 66 & 37.9 & 2 & 66.7 & 409 & 47.3 \\
\hline
\end{tabular}

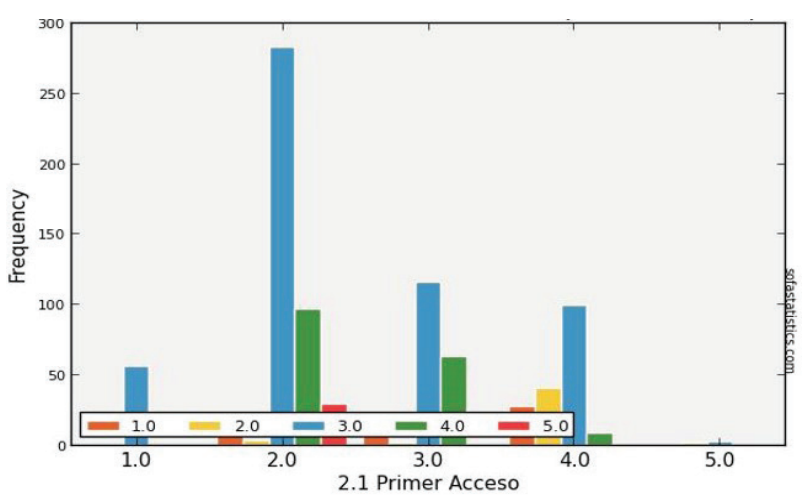

Figura 6. Frecuencia del ítem 2.1 referido a la percepción del primer acceso a webinario según sexo.

En cualquier programa académico se presentan situaciones que debe ser resueltas por los estudiantes para lograr buenos resultados en sus programas, mediante la colaboracion entre los pares y las relaciones productivas con sus similares. La utilizacion de los webinarios resultó de mucho interés para participantes mujeres (figura 7) y hombres (figura 8). Se nota que la curva representada por los datos suavizados es una normal platicurtica. 


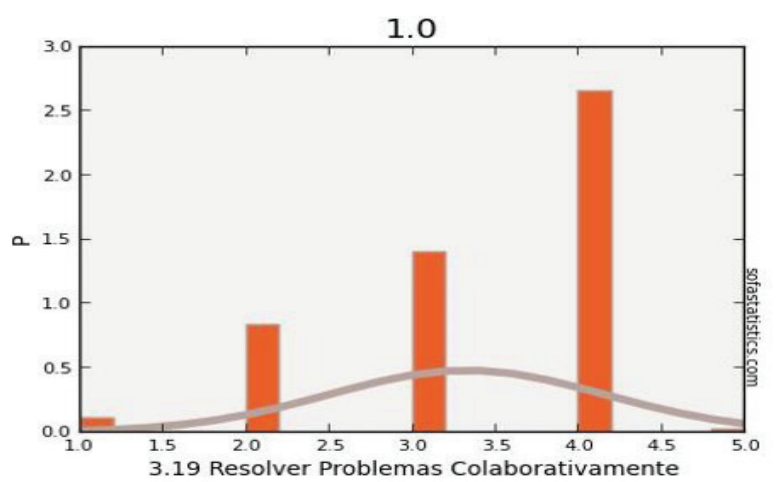

Figura 7. Histograma para representar el resultado del ítem 3,19 sobre el interés de las participantes mujeres al resolver problemas colaborativamente dentro del webinario.

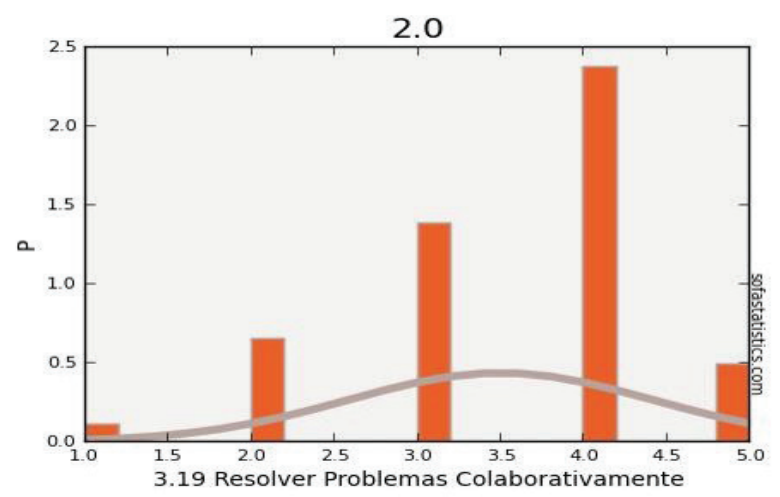

Figura 8. Histograma para representar el resultado del ítem 3,19 sobre el interés de los participantes varones al resolver problemas colaborativamente dentro del webinario.

La figura 9 muestra que los participantes menores de 25 años consideraron útiles las tutorías personales utilizando los webinarios.

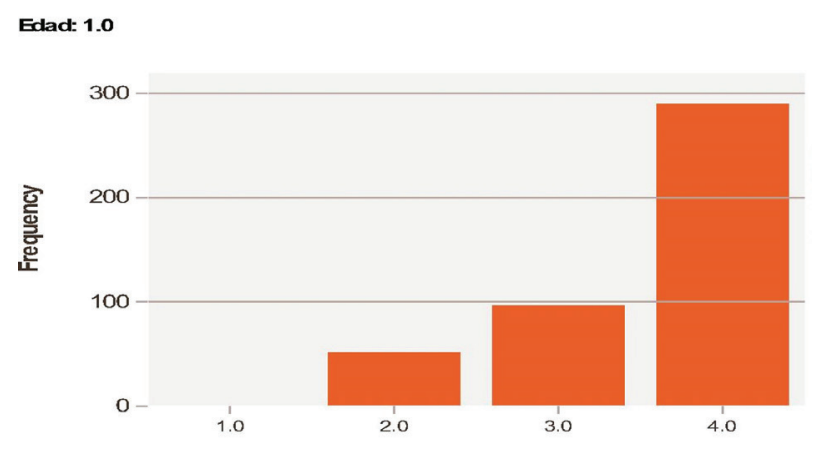

Figura 9. Frecuencia porcentual de utilidad de las tutorías personales para resolver las dudas, según edad. Edad de participantess: (1) Menor de 25 (2) 25-30 (3) 30-35 (4) Más de 35 .

La figura 10 muestra la utilidad de las tutorías personales usando los webinarios en participantes con más de 35 años.
Edad: 4.0

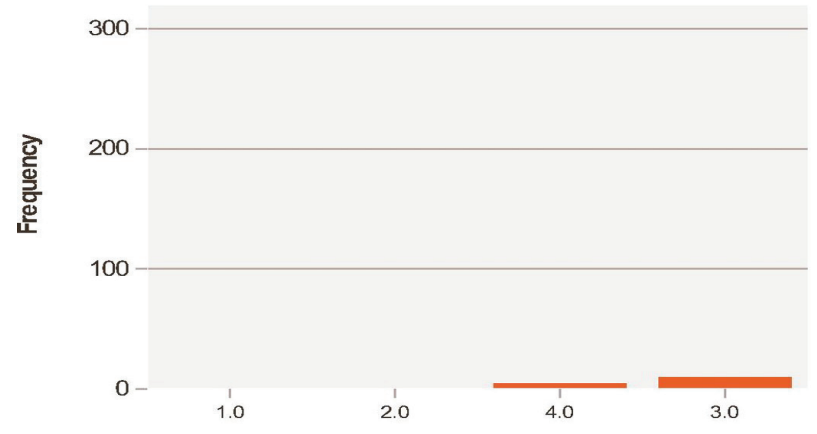

Figura 10. Frecuencia porcentual de la utilidad de las tutorías personales para resolver las dudas, según edad 4. Con participantes de edades: (1) Menos de 25 (2) 25-30 (3) 30-35 (4) Más de 35.

En la tabla 10 se muestran los resultados del primer acceso al webinario según el programa al cual pertenece el participante, y se observa que los participantes del programas de tecnología $(80.7 \%=$ 347 estudiantes) tienen la misma percepción de dificultad que los del programa de educación $(19.3 \%=83$ estudiantes).

Tabla 10. Frecuencia de los resultados del primer acceso de los estudiantes al webinario según el programa al cual pertenece.

\begin{tabular}{lrrrrrrrrrr}
\hline & \multicolumn{3}{c}{ Muy difícil } & \multicolumn{2}{c}{ Difícil } & \multicolumn{2}{c}{ Fácil } & Muy fácil & \multicolumn{2}{c}{$\begin{array}{l}\text { No sabe/ } \\
\text { No contesta }\end{array}$} \\
\cline { 2 - 12 } Programa & $f$ & $\%$ & $f$ & $\%$ & $f$ & $\%$ & $f$ & $\%$ & $f$ & $\%$ \\
\hline Tecnología & 39 & 69.6 & 347 & 80.7 & 138 & 68.7 & 134 & 77.0 & 3 & 100.0 \\
Educación & 17 & 30.4 & 83 & 19.3 & 63 & 31.3 & 40 & 23.0 & 0 & 0.0 \\
\hline
\end{tabular}

En relacion a la actividad de los tutores dentro de los webinarios, se nota que su acción no es fundamental para el desarrollo del mismo; sin embargo, sí es básica para la coordinacion de todo el proceso administrativo. Los tutores no pueden ser sustituidos, solo cambian de rol y se integran en el sistema como una figura distinta a la cotidiana. Eso le genera un reto y además genera que los estudiantes asistentes que cooperen entre pares, desarrollándose un sistema de aprendizaje colaborativo.

En la figura 11 se observa que fue bajo (49.8\%) el apoyo de los tutores en el esclarecimiento de las dudas; algo que los estudiantes consideran poco necesaria. Aunque el 43,4\% considera que es mucho o bastante el aporte del tutor. 


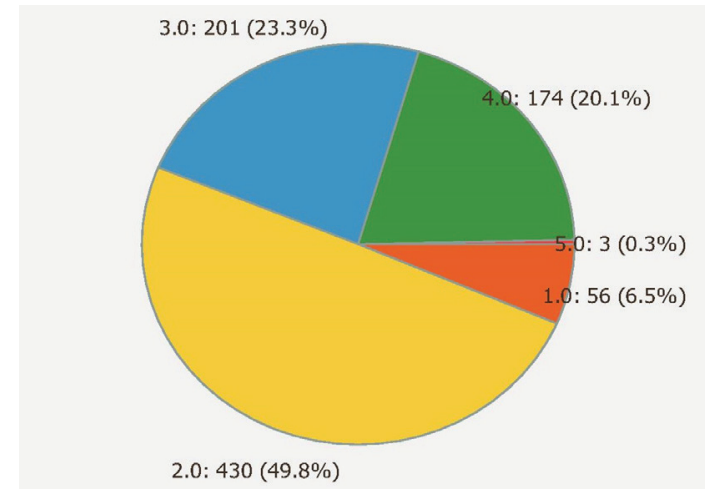

Figura 11. Frecuencia porcentual de los resultados para el ítem 3.03 referido a la superación de las dificultades de acceso y uso de webinario gracias a los tutores.

Con la utilizacion de esta innovadora herramienta, los tutores tienen el compromiso de adecuarse a estos procesos, lo que fue notado por los participantes. Las metodologías de trabajo deben cambiar y tambien la forma de mirar el rol del tutor. Como tema para una nueva investigacion queda endiente establecer si los tutores usan las técnicas apropiadas o los participantes no comprenden el nuevo rol del mismo.

Según la figura 12 , el 43,1\% de participantes sintió que los tutores no utilizaron la metodología adecuada; pero el $16,7 \%$ notaron que el trabajo de estos fue metologicamente bastante bueno. Además, se nota que más del $10 \%$ no supo evaluar este escenario.

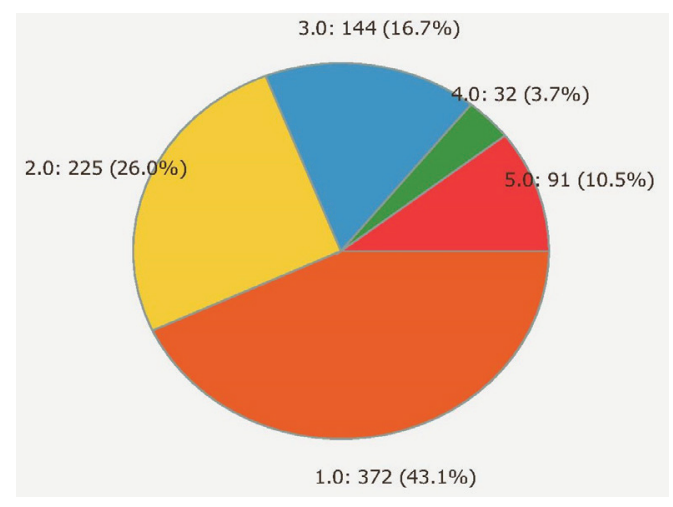

Figura 12. Frecuencia porcentual de los resultados para el ítem 3.27, referido al uso de metodologías adecuadas para fomentar la actividad creativa del webinario por parte de los tutores.

Finalmente, la figura 13 muestra el resultado de la percepción de los participantes en relación con la utilidad del webinario para aclarar dudas y realizar el trabajo colaborativo. Se advierte que todavía el
$51,9 \%$ no se siente cómodo con la utilidad de esta herramienta, lo que hace pensar en la necesidad de otro futuro trabajo para evaluar las causas.

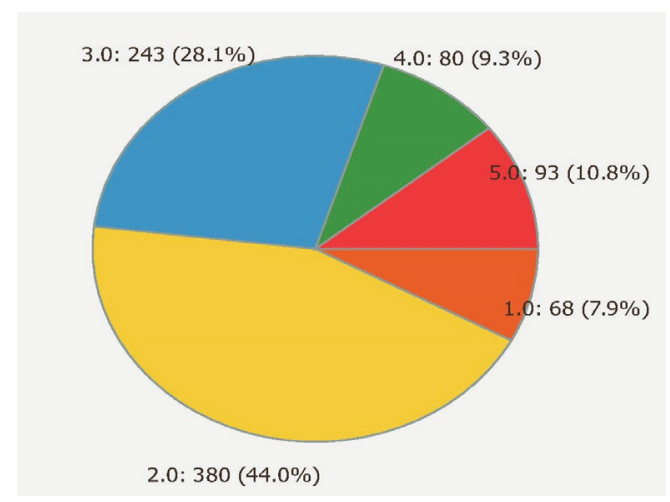

Figura 13. Frecuencia porcentual de los resultados para el ítem 3.17, relacionado con el interés que despertó la asistencia a sesiones colectivas de aclaración de dudas en nuestro espacio virtual dentro del webinario.

\section{DisCUSIÓN Y CONCLUSIONES}

Luego de hurgar dentro de los datos y develar algunos enviados por los participantes de los webinarios, se observa que la tecnología en la educación, específicamente en el contexto de esta investigación, se presenta muy interesante. Existe mucho camino por andar y mucho trabajo práctico que realizar para establecer los nuevos patrones que la educación requiere. En este trabajo se pretendió descubrir cómo un grupo de estudiantes percibían la herramienta webinario como expresión académica y de colaboración, y cómo los resultados de las investigaciones en esta área muestran las ventajas de la informática y el apego a la misma desde el mundo educativo. Pero más que eso, se buscó descubrir cómo los estudiantes se sentían cuando eran ellos quienes debían operar y utilizar esta herramienta. Quedó claro que la percepción no es tan abiertamente complaciente con la informática y específicamente con los webinarios.

Es indudable que la avalancha de mensajes e innovaciones apasiona a muchas personas. Así lo han demostrado otras investigaciones. En esta investigación, los participantes lograron integrar 
el webinario a su plan de aprendizaje personal y colaborativo y encontraron motivación para incorporarse a nuevos programas de formación. Además, dado que toda persona que se involucra en un proceso educativo y didáctico experimenta la necesidad de utilizar distintas técnicas o instrumentos para compartir información, es en la búsqueda de esas herramientas donde se inscribió esta investigación, orientada a reconocer la utilidad del webinario para los usuarios, quienes pasaron de ser simples receptores a actores generadores del seminario virtual.

Reconociendo que los elementos de mayor penetración en los sistemas educativos actuales son los relacionados con las técnicas de información y comunicación (TIC) y especialmente Internet, este estudio permitió explorar las capacidades para derribar barreras geográficas de forma práctica. Con el análisis de los resultados se pudo notar que las herramientas informáticas como el webinario son fuertes candidatos para quedarse en el ambiente académico y su utilidad sobrepasó las expectativas. También se observó un elemento que si bien no detendrá el proceso, sí dificultará el aprovechamiento de esta herramienta; esa es la percepción que los participantes tuvieron respecto a las estrategias de los tutores, donde la libertad de acción y la autonomía fue interpretada como abandono por un poco más de un tercio de los participantes. Según las entrevistas personales y según los datos, el castigo fue cercano a la mitad, así lo dejaron ver las preguntas sobre las tutorías personales, que ayudaron a despejar las dudas. Las tutorías grupales fueron de utilidad para despejar las dudas y los tutores utilizaron metodologías adecuadas para fomentar la actividad creativa del webinario; esto concuerda con investigaciones previas relacionadas con los mundos virtuales o metaverso aplicados en la educación.

La información obtenida de una informante clave dio una idea clara de los aportes de los pares dentro del aprendizaje. Se advirtió que la experiencia desarrollada fue significativa para esta estudiante. En sus palabras se pudo apreciar cómo fue aumentado su interés por la experiencia, al punto que fungió de cotutor para el resto de los participantes, guiándolos y respondiendo a las preguntas que estos le formulaban.
Los webinarios facilitan el aprendizaje colaborativo como uno de los pilares claves para la didáctica virtual. Esta es una de las premisas que justifican el uso de estas tecnologías en el campo educativo. De esta manera se podría generar propuestas contextualizadas que den respuesta a las necesidades socio-tecnológicas y educativas de un contexto social específico.

Es necesario entonces que se proponga un marco referencial para la implantación del webinario como indicador de calidad de la educación virtual y elemento que calibre los aciertos de un programa que posea elementos de enseńanza y aprendizaje asistidos por las TIC, con componentes informáticos virtualizados.

\section{REFERENCIAS BIBIOGRÁFICAS}

Area, M. et al. (2014). Webinar como estrategia de formación online: descripción y análisis de una experiencia. RELATEC. Revista Latinoamericana de Tecnología Educativa Web, 13(1), pp.1123. http://dx.doi.org/10.17398/1695-288X.13.2.11

Caballero, A. (2010). Docentes Ingeniosos 2.0 Metaversos: Una corporeidad otra. Recuperado el 10 de julio de 2015 de: http://es.slideshare.net/sybilcaballero/ docentes-ingeniosos-y-metaversossybilcaballero2010

Cabero, J. (2003). Mitos de la sociedad de la información: sus impactos en la educación. Coruña: Netbiblo. pp. 17-38.

Cabero, J. y Aguaded, I. (2013). Tecnologías y medios para la educación en la e-sociedad. ISBN 9788420678573. Recuperada el 10 de julio de 2015 de: https://dialnet.unirioja. es/servlet/articulo?codigo $=4472817$

Campo, E. y Ceballos, F. (2011). La calidad de la formación virtual en la enseñanza superior. Departamento de Automática, Universidad de Alcalá, I Congreso sobre Calidad de la Formación Virtual (CAFVIR2010) (151-158).

Catasús, M. y Ornellas, A. (2014). El docente en línea: aprender colaborando en la red. España: Editor Barcelona Universidad Abierta de Cataluña, ISBN 9788490641552.

Chardonneau, R. (2014). Google Analytics Analice el tráfico de su sitio web para mejorar los resultados (2a ed.). España: Ediciones ENI, ISBN 978-2-7460-8943-3

Del Rincón, D., Arnal, J., Latorre, A. y Sanz, A. (1995). Técnicas de investigación en Ciencias Sociales. Madrid, Es- 
pańa: Editor Dykinson.

Dirksen, J. (2015). Learning Three.js--the JavaScript 3D library for WebGL create stunning 3D graphics in your browser using the Three.js JavaScript library. Birmingham, UK: Packt Publishing.

Eouzan, G. (2013). Marketing web Definir, implementar y optimizar nuestra estrategia 2.0. Barcelona, Espańa: Ediciones ENI. ISBN 978-2-7460-8128-4

Esté, A. (2006). Mejoramiento de la formación básica y modernización de los sistemas de formación docente. Ponencia presentada en el Seminario Taller Descentralización: Una estrategias para el desarrollo educativo, Maracaibo.

Fox, D. (1981). El proceso de investigación en educación. Pamplona: Editorial Eunsa. pp 45-59.

Friendly, M. y Meyer, D. (2015). Discrete Data Analysis with R: Visualization and Modeling Techniques for Categorical and Count Data. Editorial Chapman and Hall/CRC. ISBN 9781498725835

Horachek, D. (2014). Creating e-learning games with Unity, develop your own 3D e-learning game using gamification, systems design, and gameplay programming techniques. Editor Birmingham. ISBN 9781849693424

Ledesma, R., Molina, J. y Valero, P. (2002). Análisis de consistencia interna mediante Alfa de Cronbach: Un programa basado en gráficos dinámicos. Revista PSICO-USF, vol. 7, pp. 143-152.

Nof, S. (2015). Revolutionizing Collaboration through e-Work, e-Business, and e-Service. Publicación Springer Berlin Heidelberg. ISBN 9783662457771

Marradi, A. (2012). Metodología de las Ciencias Sociales (2a ed.). Buenos Aires, Argentina: CENGAGE, Recuperado de https://issuu.com/cengagelatam/docs/ metodologia_de_ las_ciencias_sociales_marradi

Okuda, M. (2005). Métodos en investigación cualitativa: triangulación. En: Scielo, Revista Colombiana de Psiquiatría. 34 (1). ISSN 0034-7450. Recuperado el 10 de julio de 2015 de: http://www.scielo.org.co/scielo.php? script=sci_arttext\&pid=S0034-74502005000100008

Parisi, T. (2012). WebGL up and running. Sebastopol, O’Reilly Media. ISBN 9781449323578

Peña, J. y Ortega, J. (2012). Los metaversos como micro ciudades digitales: experiencias de movilidad virtual en el espacio académico américa latina-caribe-unión europea realizadas desde la red euro-iberoamericana ecaeva-virtualcue. Madrid: Editorial Icono14. Presentado en II Congreso Internacional Ciudades Creativas Universidad Complutense de Madrid.

Peña, J. (2012). Simulación digital de metaversos para situaciones educativas: una propuesta-didáctica para el máster iberoamericano en educación a distancia en entornos virtuales. Universidad Pedagógica Libertador: Maracay

Pérez, A. (2011). La integración curricular del cine digital en la formación inicial de los maestros: perspectivas didácticas y creativas. Tesis doctoral inédita, Universidad de Granada:
España.

Pérez, M. (2011). Innovación metodológica y Espacio Europeo de Educación Superior. España: Editor Dykinson. ISBN 9788498498295. Presentado en el Congreso CLED 2010 .

Quintero, M. (2014). El uso de Webinar en las instituciones educativas. Blog. Recuperado el 01 de marzo de 2015 de: https://prezi.com/mbetxexmhsmu/el-uso-de-webinar-en-las-instituciones-educativas/

Santnerand, T. y Duffy, D. (1989). The Statistical Analysis of Discrete Data. Springer: Verlag.

Souto, E. (2010). Educación, democracia y ciudadanía. Madrid: Editor Dykinson. ISBN 9788499820347.

Vázquez, M. (2011). Webinar: seminarios virtuales. Fundación EROSKI. Recuperado el 23 de septiembre de 2013 de: http://www.consumer.es/web/es/educacion/ otras_formaciones/2011/10/30/204271.php 